\title{
WEYL'S THEOREM FOR OPERATOR MATRICES
}

\author{
Woo Young Lee ${ }^{1}$
}

\begin{abstract}
"Weyl's theorem holds" for an operator when the complement in the spectrum of the "Weyl" spectrum" coincides with the isolated points of the spectrum which are eigenvalues of finite multiplicity. By comparison "Browder's theorem holds" for an operator when the complement in the spectrum of the Weyl spectrum coincides with Riesz points. Weyl's theorem and Browder's theorem are liable to fail for $2 \times 2$ operator matrices. In this paper we explore how Weyl's theorem and Browder's theorem survive for $2 \times 2$ operator matrices on the Hilbert space.
\end{abstract}

\section{Introduction}

Weyl's theorem for an operator says that the complement in the spectrum of the Weyl spectrum coincides with the isolated points of the spectrum which are eigenvalues of finite multiplicity. H. Weyl ([22]) discovered that this property holds for hermitian operators and it has been extended from hermitian operators to hyponormal operators and to Toeplitz operators by L. Coburn ([5]), and to several classes of operators including seminormal operators by S. Berberian $([1],[2])$. Recently Weyl's theorem under the "small" perturbations has been considered in [14] and [15]. But Weyl's theorem is liable to fail for $2 \times 2$ (even diagonal) operator matrices even though Weyl's theorem holds for the entries in the operator matrices. In this paper we consider Weyl's theorem and the less restrictive "Browder's theorem" for $2 \times 2$ operator matrices.

Let $\mathcal{H}$ and $\mathcal{K}$ be Hilbert spaces, let $\mathcal{L}(\mathcal{H}, \mathcal{K})$ denote the set of bounded linear operators from $\mathcal{H}$ to $\mathcal{K}$, and abbreviate $\mathcal{L}(\mathcal{H}, \mathcal{H})$ to $\mathcal{L}(\mathcal{H})$. If $A \in \mathcal{L}(\mathcal{H})$ is a Fredholm operator, that is, if $A$ has finite dimensional null space and its range of finite co-dimension, then the index of $A$, denoted ind $A$, is given by

$$
\text { ind } A=\operatorname{dim} A^{-1}(0)-\operatorname{dim} A(\mathcal{H})^{\perp} .
$$

An operator $A \in \mathcal{L}(\mathcal{H})$ is called Weyl if it is Fredholm of index zero and is called Browder if it is Fredholm "of finite ascent and descent": equivalently ([12, Theorem 7.9.3]) if $A$

\footnotetext{
${ }^{1}$ Supported in part by BSRI-97-1420 and KOSEF 94-0701-02-01-3.
} 
is Fredholm and $A-\lambda I$ is invertible for sufficiently small $\lambda \neq 0$ in $\mathbb{C}$. The essential spectrum $\sigma_{e}(A)$, the Weyl spectrum $\omega(A)$ and the Browder's spectrum $\sigma_{b}(A)$ of $A \in \mathcal{L}(\mathcal{H})$ are defined by

$$
\begin{aligned}
\sigma_{e}(A) & =\{\lambda \in \mathbb{C}: A-\lambda I \text { is not Fredholm }\} \\
\omega(A) & =\{\lambda \in \mathbb{C}: A-\lambda I \text { is not Weyl }\} \\
\sigma_{b}(A) & =\{\lambda \in \mathbb{C}: A-\lambda I \text { is not Browder }\}:
\end{aligned}
$$

evidently

$$
\sigma_{e}(A) \subseteq \omega(A) \subseteq \sigma_{b}(A)=\sigma_{e}(A) \cup \operatorname{acc} \sigma(A),
$$

where we write acc $\mathbf{K}$ for the accumulation points of $\mathbf{K} \in \mathbb{C}$ and $\sigma(A)$ for the ordinary spectrum of $A$. If we write iso $\mathbf{K}=\mathbf{K} \backslash \operatorname{acc} \mathbf{K}$ and

$$
\pi_{00}(A):=\left\{\lambda \in \text { iso } \sigma(A): 0<\operatorname{dim}(A-\lambda I)^{-1}(0)<\infty\right\}
$$

for the isolated eigenvalues of finite multiplicity, and ([12, (9.8.3.4)])

$$
p_{00}(A):=\sigma(A) \backslash \sigma_{b}(A)
$$

for the Riesz points of $A$, then

$$
\text { iso } \sigma(A) \backslash \sigma_{e}(A)=\text { iso } \sigma(A) \backslash \omega(A)=p_{00}(A) \subseteq \pi_{00}(A) .
$$

The authors of [14] and [15] use the notation of (0.1) for the concept of (0.2). We say that Weyl's theorem holds for $A \in \mathcal{L}(\mathcal{H})$ if

$$
\sigma(A) \backslash \omega(A)=\pi_{00}(A),
$$

and ([14, Definition 1]) that Browder's theorem holds for $A$ if

$$
\sigma(A) \backslash \omega(A)=p_{00}(A) .
$$

Evidently "Weyl's theorem" implies "Browder's theorem". Recall $([2],[19])$ that an operator $A \in \mathcal{L}(\mathcal{H})$ is called isoloid if every isolated point of $\sigma(A)$ is an eigenvalue of $A$.

\section{Weyl's theorem for $2 \times 2$ skew-diagonal operator matrices}

Weyl's theorem may or may not hold for a direct sum of operators for which Weyl's theorem holds. For example, if $U$ is the unilateral shift on $\ell_{2}$ then Weyl's theorem holds for both $U$ and $U^{*}$, while it does not hold for $U \oplus U^{*}$. But it was known ([4],[16]) that if $A$ and $B$ are isoloid and if Weyl's theorem holds for $A$ and $B$ then

$$
\text { Weyl's theorem holds for }\left(\begin{array}{cc}
A & 0 \\
0 & B
\end{array}\right) \Longleftrightarrow \omega\left(\begin{array}{cc}
A & 0 \\
0 & B
\end{array}\right)=\omega(A) \cup \omega(B) \text {. }
$$

Also the equivalence (1.0.1) holds for Browder's theorem without the isoloid assumption (cf.[14]). In this section we consider Weyl's theorem for the $2 \times 2$ skew-diagonal operator matrix of the form $\left(\begin{array}{ll}0 & A \\ B & 0\end{array}\right)$. Weyl's theorem for the skew-diagonal matrices is more delicate in comparison with the diagonal matrices. We begin with: 
Lemma 1.1. If $A \in \mathcal{L}(\mathcal{H}, \mathcal{K})$ and $B \in \mathcal{L}(\mathcal{K}, \mathcal{H})$, then the non-zero elements of $\varpi(A B)$ and $\varpi(B A)$ are the same for each $\varpi \in\left\{\sigma, \sigma_{e}, \omega\right\}$.

Proof. Remember (cf. [9, p.38]) that if $\lambda \neq 0$ then

$$
\left(\begin{array}{cc}
A B-\lambda I & 0 \\
0 & I
\end{array}\right)=F(\lambda)\left(\begin{array}{cc}
B A-\lambda I & 0 \\
0 & I
\end{array}\right) E(\lambda)
$$

where $E(\lambda)$ and $F(\lambda)$ are both invertible for each $\lambda \neq 0$. It thus follows from (1.1.1) that if $\lambda \neq 0$

$$
(A B-\lambda I)^{-1}(0) \cong(B A-\lambda I)^{-1}(0) \quad \text { and } \quad((A B-\lambda I)(\mathcal{K}))^{\perp} \cong((B A-\lambda I)(\mathcal{H}))^{\perp},
$$

which gives the result.

Although $\sigma(A B)=\sigma(B A)$, we need not expect that $\omega(A B)=\omega(B A)$. To see this, let $\operatorname{dim} \mathcal{X}<\infty$ and $\mathcal{Y}=\ell_{2}$, let $S, T: \mathcal{Y} \rightarrow \mathcal{Y}$ be defined by

$$
S\left(x_{1}, x_{2}, \cdots\right)=\left(0, x_{1}, 0, x_{2}, 0, x_{3}, \cdots\right) \text { and } T\left(x_{1}, x_{2}, \cdots\right)=\left(x_{2}, x_{4}, x_{6}, \cdots\right),
$$

and put $A=0_{\mathcal{X}} \oplus S$ and $B=0_{\mathcal{X}} \oplus T$, then $\sigma(A B)=\sigma(B A)=\{0,1\}$, while $\omega(A B)=$ $\{0,1\} \neq \omega(B A)=\{1\}$. However one might be tempted to guess that if $\sigma(A B)=\sigma(B A)$ and if in addition $\sigma(A B)$ is connected, then $\omega(A B)=\omega(B A)$. But this guess is also wrong. For an example, if on $\ell_{2} \otimes \ell_{2}$

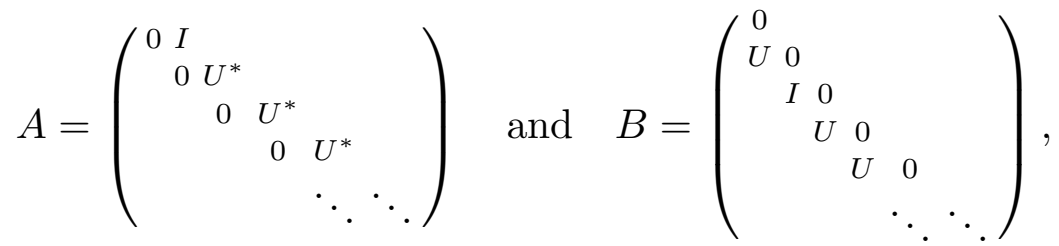

where $U$ is the unilateral shift on $\ell_{2}$, then a straightforward calculation shows that $\sigma(A B)=$ $\sigma(B A)=\mathbb{D}$, while $\omega(A B)=\mathbb{T} \neq \omega(B A)=\mathbb{T} \cup\{0\}$, where $\mathbb{D}$ and $\mathbb{T}$ denote the closed unit disk and the unit circle, respectively.

Lemma 1.2. If $A \in \mathcal{L}(\mathcal{K}, \mathcal{H})$ and $B \in \mathcal{L}(\mathcal{H}, \mathcal{K})$, then there is equality

$$
\omega\left(\begin{array}{cc}
A B & 0 \\
0 & B A
\end{array}\right)=\omega(A B) \cup \omega(B A) \text {. }
$$

Hence, in particular, if $A B$ and $B A$ are isoloid and if Weyl's theorem holds for $A B$ and $B A$ then Weyl's theorem holds for $\left(\begin{array}{cc}A B & 0 \\ 0 & B A\end{array}\right)$.

Proof. The inclusion " $\subseteq$ " in (1.2.1) follows from the fact that the index of a direct sum is the sum of the indices. For the inclusion " $\supseteq$ ", suppose that $\left(\begin{array}{cc}A B-\lambda I & 0 \\ 0 & B A-\lambda I\end{array}\right)$ is Weyl. Then $A B-\lambda I$ and $B A-\lambda I$ are both Fredholm, and ind $(A B-\lambda I)+$ ind $(B A-\lambda I)=0$. But if $\lambda \neq 0$ then by Lemma 1.1, ind $(A B-\lambda I)=$ ind $(B A-\lambda I)$, so that we must have that $A B-\lambda I$ and $B A-\lambda I$ are both Weyl. If instead $\lambda=0$ then since $A B$ and $B A$ are both Fredholm it follows from the continuity of the index that for sufficiently small $|\mu|$ $(\mu \neq 0)$,

$$
\text { ind }(A B)=\operatorname{ind}(A B-\mu I)=\operatorname{ind}(B A-\mu I)=\operatorname{ind}(B A) \text {, }
$$

which also forces that $A B$ and $B A$ are both Weyl. This proves (1.2.1). The second assertion follows at once from (1.0.1). 
Example 1.3. (a) If $\mathcal{H}=\mathcal{K}$ in Lemma 1.2, one might expect to replace the condition "Weyl's theorem holds for $A B$ and $B A$ " by the condition "Weyl's theorem holds for $A$ and $B$ ". But this is not the case: for example, Weyl's theorem may fail for $T^{2}$ when it holds for the operator $T$ (see [19, Example 1]).

(b) Since

$$
\left(\begin{array}{cc}
0 & A \\
B & 0
\end{array}\right)^{2}=\left(\begin{array}{cc}
A B & 0 \\
0 & B A
\end{array}\right)
$$

one might also expect that "Weyl's theorem for $\left(\begin{array}{cc}0 & A \\ B & 0\end{array}\right)$ " is inherited from "Weyl's theorem for $\left(\begin{array}{cc}A B & 0 \\ 0 & B A\end{array}\right)$ ". But in general Weyl's theorem need not be transmitted from the square of the operator $T$ to $T$. For example, if $U$ is the unilateral shift on $\ell_{2}$ and $K: \ell_{2} \rightarrow \ell_{2}$ is defined by

$$
K\left(x_{1}, x_{2}, \cdots\right)=\left(\frac{1}{2} x_{2}, \frac{1}{3} x_{3}, \cdots\right)
$$

put on $\ell_{2} \oplus \ell_{2}$

$$
T=\left(\begin{array}{cc}
U+1 & 0 \\
0 & K-1
\end{array}\right):
$$

then $\sigma(T)=\omega(T)=\{z:|z-1| \leq 1\} \cup\{-1\}$ and $\pi_{00}(T)=\{-1\}$, while

$$
\sigma\left(T^{2}\right)=\omega\left(T^{2}\right)=\left\{r e^{i \theta}: r \leq 2(1+\cos \theta)\right\} \quad \text { and } \quad \pi_{00}\left(T^{2}\right)=\emptyset,
$$

which says that Weyl's theorem holds for $T^{2}$, but fails for $T$.

(c) In general "Weyl's theorem holds for $A B$ " does not imply "Weyl's theorem holds for $B A$ ". For example if the operators $K, S$ and $T$ on $\ell_{2}$ are defined as in (1.3.1) and (1.1.2), put on $\ell_{2} \oplus \ell_{2}$

$$
A=K \oplus S \quad \text { and } \quad B=1 \oplus T:
$$

then $\sigma(A B)=\omega(A B)=\sigma(B A)=\omega(B A)=\{0,1\}$, while $\pi_{00}(A B)=\emptyset \neq \pi_{00}(B A)=\{0\}$.

In spite of Example 1.3 (b), we have:

Theorem 1.4. Let $A \in \mathcal{L}(\mathcal{K}, \mathcal{H})$ and $B \in \mathcal{L}(\mathcal{H}, \mathcal{K})$ be such that $A B$ and $B A$ are isoloid. If Weyl's theorem holds for $A B$ and $B A$ then it holds for $\left(\begin{array}{cc}0 & A \\ B & 0\end{array}\right)$.

Proof. By an argument of $[15,(3.10) ;(4.3)]$, we have that with no restriction on either $A$ or $B$,

$$
\varpi\left(\begin{array}{cc}
0 & A \\
B & 0
\end{array}\right)=\sqrt{\varpi(A B) \cup \varpi(B A)} \quad \text { for each } \varpi \in\left\{\sigma, \sigma_{e}, \omega\right\},
$$

where $\sqrt{\mathbf{K}}$ denotes the set of square roots of complex numbers in $\mathbf{K} \subseteq \mathbb{C}$. Thus if Weyl's theorem holds for $A B$ and $B A$ then by Lemma 1.2, there is equality

$$
\begin{aligned}
\sigma\left(\begin{array}{cc}
0 & A \\
B & 0
\end{array}\right) \backslash \omega\left(\begin{array}{cc}
0 & A \\
B & 0
\end{array}\right) & =\sqrt{\sigma(A B) \cup \sigma(B A) \backslash \sqrt{\omega(A B) \cup \omega(B A)}} \\
& =\sqrt{(\sigma(A B) \cup \sigma(B A)) \backslash(\omega(A B) \cup \omega(B A))} \\
& =\sqrt{\sigma\left(\begin{array}{cc}
A B & 0 \\
0 & B A
\end{array}\right) \backslash \omega\left(\begin{array}{cc}
A B & 0 \\
0 & B A
\end{array}\right)} \\
& =\sqrt{\pi_{00}\left(\begin{array}{cc}
A B & 0 \\
0 & B A
\end{array}\right) .}
\end{aligned}
$$


Now it will be shown that

$$
\sqrt{\pi_{00}\left(\begin{array}{cc}
A B & 0 \\
0 & B A
\end{array}\right)}=\pi_{00}\left(\begin{array}{cc}
0 & A \\
B & 0
\end{array}\right)
$$

But since, in view of (1.4.1), $\sigma\left(\begin{array}{cc}0 & A \\ B & 0\end{array}\right)$ is symmetric with respect to the origin, it follows from the spectral mapping theorem that

$$
\sqrt{\text { iso } \sigma\left(\begin{array}{cc}
A B & 0 \\
0 & B A
\end{array}\right)}=\sqrt{\text { iso }\left(\sigma\left(\begin{array}{cc}
0 & A \\
B & 0
\end{array}\right)\right)^{2}}=\operatorname{iso} \sigma\left(\begin{array}{cc}
0 & A \\
B & 0
\end{array}\right) \text {. }
$$

Thus for (1.4.2) it suffices to show that, for any $\mu \in \mathbb{C}$,

$$
0<\operatorname{dim}\left(\begin{array}{cc}
A B-\mu I & 0 \\
0 & B A-\mu I
\end{array}\right)^{-1}\left(\begin{array}{l}
0 \\
0
\end{array}\right)<\infty \Longleftrightarrow 0<\operatorname{dim}\left(\begin{array}{cc}
-\sqrt{\mu} I & A \\
B & -\sqrt{\mu} I
\end{array}\right)^{-1}\left(\begin{array}{l}
0 \\
0
\end{array}\right)<\infty
$$

If $\mu=0$, then (1.4.3) follows from the observation

$$
0<\operatorname{dim}\left(A^{-1}(0) \oplus B^{-1}(0)\right)<\infty \Longleftrightarrow 0<\operatorname{dim}\left((A B)^{-1}(0) \oplus(B A)^{-1}(0)\right)<\infty
$$

If instead $\mu \neq 0$, then (1.4.3) follows from the observation

$$
\begin{aligned}
& \bigvee\left\{\left(x, \frac{1}{\sqrt{\mu}} B x\right): x \in(A B-\mu I)^{-1}(0)\right\} \bigcup \bigvee\left\{\left(\frac{1}{\sqrt{\mu}} A y, y\right): y \in(B A-\mu I)^{-1}(0)\right\} \\
& \subseteq\left(\begin{array}{cc}
-\sqrt{\mu} I & A \\
B & -\sqrt{\mu} I
\end{array}\right)^{-1}\left(\begin{array}{c}
0 \\
0
\end{array}\right) \subseteq(A B-\mu I)^{-1}(0) \oplus(B A-\mu I)^{-1}(0)
\end{aligned}
$$

where $\bigvee G$ denotes the closed linear span of $G$. This proves (1.4.2) and completes the proof.

Corollary 1.5. Lemma 1.2 and Theorem 1.4 remain true with Browder's theorem in place of Weyl's theorem.

Proof. The same argument as Lemma 1.2 and Theorem 1.4 gives the result.

Note that $A^{2}$ may not be hyponormal when $A$ is hyponormal ([10, Problem 209]). In spite of it, if $A$ is hyponormal then Weyl's theorem holds for $f(A)$, where $f \in H(\sigma(A)):=$ the set of analytic functions on an open neighborhood of $\sigma(A)$ (cf. [17, Theorem 2];[21, Theorem 3.6]). Thus we have:

Corollary 1.6. If $A$ is hyponormal then Weyl's theorem holds for $\left(\begin{array}{cc}0 & f(A) \\ g(A) & 0\end{array}\right)$ for every $f, g \in H(\sigma(A))$.

Proof. It is easy to show that if $A$ is isoloid then $h(A)$ is also isoloid for every $h \in H(\sigma(A))$. Thus the result follows at once from Theorem 1.4. 
The assumption of Theorem 1.4 can easily be satisfied by "Toeplitz operators": recall ([6]) that if $P$ denotes the orthogonal projection from $L^{2}(\mathbb{T})$ onto the Hardy space $H^{2}(\mathbb{T})$ then for every $\varphi \in L^{\infty}(\mathbb{T})$, the operator $T_{\varphi}$ on $H^{2}(\mathbb{T})$ defined by

$$
T_{\varphi} g=P(\varphi g) \quad \text { for each } g \in H^{2}(\mathbb{T})
$$

is called the Toeplitz operator with symbol $\varphi$. It is familiar that the spectrum of a Toeplitz operator is always connected ([23]), and that the spectrum and the Weyl spectrum coincide, and hence Weyl's theorem holds for every Toeplitz operator ([5]). Evidently, every Toeplitz operator is isoloid. If either $\varphi$ is analytic (i.e., $\varphi \in H^{\infty}(\mathbb{T}):=L^{\infty} \cap H^{2}(\mathbb{T})$ ) or $\psi$ is coanalytic (i.e., $\bar{\psi} \in H^{\infty}(\mathbb{T})$ ) then $T_{\psi} T_{\varphi}=T_{\psi \varphi}$ (cf. [6, Proposition 7.5]). Also if we write $C(\mathbb{T})$ for the set of all continuous functions on $\mathbb{T}$ then, for every $\varphi \in C(\mathbb{T})$, (cf. $[6$, Proposition 7.22])

$$
T_{\varphi} T_{\psi}-T_{\varphi \psi} \text { and } T_{\psi} T_{\varphi}-T_{\psi \varphi} \text { are compact operators for every } \psi \in L^{\infty}(\mathbb{T}) \text {. }
$$

We then have:

Example 1.7. If $\varphi$ is either analytic or coanalytic and if $\psi \in C(\mathbb{T})$ then Weyl's theorem holds for $\left(\begin{array}{cc}0 & T_{\varphi} \\ T_{\psi} & 0\end{array}\right)$.

Proof. Suppose that $\varphi \in H^{\infty}(\mathbb{T})$. Then $T_{\psi} T_{\varphi}=T_{\psi \varphi}$, and hence $T_{\psi} T_{\varphi}$ is isoloid and Weyl's theorem holds for $T_{\psi} T_{\varphi}$. Therefore, in view of Theorem 1.4, it suffices to prove that $T_{\varphi} T_{\psi}$ is isoloid and Weyl's theorem holds for $T_{\varphi} T_{\psi}$. Suppose that $\psi \in C(\mathbb{T})$. If $\sigma\left(T_{\varphi} T_{\psi}\right)=\sigma\left(T_{\psi} T_{\varphi}\right)$ then by (1.6.1),

$$
\omega\left(T_{\varphi} T_{\psi}\right)=\omega\left(T_{\varphi \psi}\right)=\sigma\left(T_{\psi \varphi}\right)=\sigma\left(T_{\psi} T_{\varphi}\right)=\sigma\left(T_{\varphi} T_{\psi}\right),
$$

because the Weyl spectrum is invariant under the compact perturbations. Since $\sigma\left(T_{\varphi} T_{\psi}\right)=$ $\sigma\left(T_{\psi} T_{\varphi}\right)$ and hence $\sigma\left(T_{\varphi} T_{\psi}\right)$ is connected, (1.7.1) implies that $T_{\varphi} T_{\psi}$ is isoloid and Weyl's theorem holds for $T_{\varphi} T_{\psi}$. If instead $\sigma\left(T_{\varphi} T_{\psi}\right) \neq \sigma\left(T_{\psi} T_{\varphi}\right)$ then since $\sigma\left(T_{\psi} T_{\varphi}\right)$ is connected, it follows from Lemma 1.1 that

$$
0 \notin \sigma\left(T_{\psi} T_{\varphi}\right) \quad \text { and } \quad 0 \in \text { iso } \sigma\left(T_{\varphi} T_{\psi}\right) .
$$

But then $T_{\psi} T_{\varphi}$ is invertible and hence, by (1.6.1), $T_{\varphi} T_{\psi}$ is Weyl but not invertible, which implies that $0 \in \pi_{00}\left(T_{\varphi} T_{\psi}\right)$ and hence $T_{\varphi} T_{\psi}$ is isoloid. Therefore there is equality

$$
\begin{aligned}
\sigma\left(T_{\varphi} T_{\psi}\right) \backslash \omega\left(T_{\varphi} T_{\psi}\right) & =\left(\sigma\left(T_{\psi} T_{\varphi}\right) \backslash \omega\left(T_{\psi} T_{\varphi}\right)\right) \cup\{0\} \\
& =\pi_{00}\left(T_{\psi} T_{\varphi}\right) \cup\{0\} \\
& =\pi_{00}\left(T_{\varphi} T_{\psi}\right),
\end{aligned}
$$

which says that Weyl's theorem holds for $T_{\varphi} T_{\psi}$. The argument for the case of the coanalytic symbol $\varphi$ is the same. 
Example 1.8. (a) If $\varphi$ and $\psi$ are in $C(\mathbb{T})$, then Weyl's theorem need not hold for $\left(\begin{array}{cc}0 & T_{\varphi} \\ T_{\psi} & 0\end{array}\right)$. For example if $\varphi$ is defined by

$$
\varphi\left(e^{i \theta}\right)= \begin{cases}-e^{2 i \theta}+1 & (0 \leq \theta \leq \pi) \\ e^{-2 i \theta}-1 & (\pi \leq \theta \leq 2 \pi)\end{cases}
$$

then a straightforward calculation shows (cf. [8, Example 3.3])

$$
\sigma\left(\begin{array}{cc}
0 & T_{\varphi} \\
T_{\varphi} & 0
\end{array}\right)=\sqrt{\sigma\left(T_{\varphi}^{2}\right)}=\sqrt{\sigma\left(T_{\varphi}\right)^{2}}=\sqrt{\left\{r e^{i \theta}: r \leq 2(1+\cos \theta)\right\}}
$$

and

$$
\omega\left(\begin{array}{cc}
0 & T_{\varphi} \\
T_{\varphi} & 0
\end{array}\right)=\sqrt{\omega\left(T_{\varphi}^{2}\right)}=\sqrt{\omega\left(T_{\varphi^{2}}\right)}=\sqrt{\left\{r e^{i \theta}: r=2(1+\cos \theta)\right\}}
$$

which implies that Weyl's theorem (even Browder's theorem) does not hold for $\left(\begin{array}{cc}0 & T_{\varphi} \\ T_{\varphi} & 0\end{array}\right)$.

(b) As we noticed, if $U$ is the unilateral shift on $\ell_{2}$ then Weyl's theorem fails for $\left(\begin{array}{cc}U & 0 \\ 0 & U^{*}\end{array}\right)$. But Example 1.7 guarantees that Weyl's theorem holds for $\left(\begin{array}{cc}0 & U \\ U^{*} & 0\end{array}\right)$.

\section{Weyl's theorem for $2 \times 2$ upper triangular operator matrices}

Weyl's theorem and Browder's theorem are transmitted from $T \in \mathcal{L}(\mathcal{H})$ to $T+K$ for commuting nilpotents $K \in \mathcal{L}(\mathcal{H})$ (cf. [14],[19]). But this may fail if $K$ is not assumed to commute with $T$ even if $K$ is both compact and nilpotent: for example, consider $T=\left(\begin{array}{cc}U & 1-U U^{*} \\ 0 & U^{*}\end{array}\right)$ and $K=\left(\begin{array}{cc}0 & 1-U U^{*} \\ 0 & 0\end{array}\right)$ (cf. [14, Example 12]). In this section we consider the following question: if Weyl's theorem holds for $\left(\begin{array}{cc}A & 0 \\ 0 & B\end{array}\right)$, when does it hold for $\left(\begin{array}{cc}A & C \\ 0 & B\end{array}\right)$ ? To state the main result we need the concept of the "spectral picture" ([3],[20]) of the operator $A \in \mathcal{L}(\mathcal{H})$, denoted $\mathcal{S P}(A)$, which consists of the set $\sigma_{e}(A)$, the collection of holes and pseudoholes in $\sigma_{e}(A)$, and the indices associated with these holes and pseudoholes.

When $A \in \mathcal{L}(\mathcal{H})$ and $B \in \mathcal{L}(\mathcal{K})$ are given we denote by $M_{C}$ an operator acting on $\mathcal{H} \oplus \mathcal{K}$ of the form

$$
M_{C}=\left(\begin{array}{cc}
A & C \\
0 & B
\end{array}\right),
$$

where $C \in \mathcal{L}(\mathcal{K}, \mathcal{H})$. It was shown in [16] that

$$
\omega\left(M_{C}\right) \subseteq \omega\left(\begin{array}{cc}
A & 0 \\
0 & B
\end{array}\right) \subseteq \omega(A) \cup \omega(B)
$$

In [7] and [16], the structures of spectra and Weyl spectra of $M_{C}$ were considered in detail.

Lemma $2.1([\mathbf{7}],[\mathbf{1 6}])$. For a given pair $(A, B)$ of operators there is equality, for every $C \in \mathcal{L}(\mathcal{K}, \mathcal{H})$

$$
\varpi(A) \cup \varpi(B)=\varpi\left(M_{C}\right) \cup \mathfrak{S} \quad \text { with } \varpi \in\{\sigma, \omega\},
$$

where $\mathfrak{S}$ is the union of certain of the holes in $\varpi\left(M_{C}\right)$ which happen to be subsets of $\varpi(A) \cap \varpi(B)$. 
Lemma 2.2. If either $\mathcal{S P}(A)$ or $\mathcal{S P}(B)$ has no pseudoholes then, for every $C \in \mathcal{L}(\mathcal{K}, \mathcal{H})$, we have:

(a) $\omega\left(\begin{array}{ll}A & 0 \\ 0 & B\end{array}\right)=\omega\left(M_{C}\right)$.

(b) $\sigma(A) \cup \sigma(B)=\sigma\left(M_{C}\right) \cup \mathfrak{S}$, where $\mathfrak{S}$ is the union of certain of the holes in $\sigma\left(M_{C}\right)$ which happen to be subsets of $(\sigma(A) \cap \sigma(B)) \backslash \omega\left(\begin{array}{cc}A & 0 \\ 0 & B\end{array}\right)$.

Proof. The proof of the statement (a) is known from [16, Corollary 3] and the statement (b) follows at once from Lemma 2.1 and the statement (a).

Although the passage from $\sigma\left(M_{C}\right)$ to $\sigma\left(\begin{array}{cc}A & 0 \\ 0 & B\end{array}\right)$ is the filling in certain of the holes in $\sigma\left(M_{C}\right)$, we cannot expect that iso $\sigma\left(\begin{array}{cc}A & 0 \\ 0 & B\end{array}\right)=$ iso $\sigma\left(M_{C}\right)$ even when both $\mathcal{S P}(A)$ and $\mathcal{S P}(B)$ have no pseudoholes. For example if on $\ell_{2} \oplus \ell_{2}$

$$
A=U \oplus 0, \quad B=U^{*} \oplus 0, \quad \text { and } \quad C=\left(1-U U^{*}\right) \oplus 0,
$$

where $U$ is the unilateral shift on $\ell_{2}$, then $\sigma\left(\begin{array}{cc}A & 0 \\ 0 & B\end{array}\right)=\mathbb{D}$ and $\sigma\left(\begin{array}{cc}A & C \\ 0 & B\end{array}\right)=\mathbb{T} \cup\{0\}$, while $\omega\left(\begin{array}{cc}A & 0 \\ 0 & B\end{array}\right)=\mathbb{T} \cup\{0\}=\omega\left(\begin{array}{cc}A & C \\ 0 & B\end{array}\right)$.

Theorem 2.3. If either $\mathcal{S P}(A)$ or $\mathcal{S P}(B)$ has no pseudoholes then for every $C \in \mathcal{L}(\mathcal{K}, \mathcal{H})$,

Browder's theorem holds for $\left(\begin{array}{cc}A & 0 \\ 0 & B\end{array}\right) \Longrightarrow$ Browder's theorem holds for $\left(\begin{array}{cc}A & C \\ 0 & B\end{array}\right)$.

Proof. By assumption we have that $\sigma\left(\begin{array}{cc}A & 0 \\ 0 & B\end{array}\right) \backslash \omega\left(\begin{array}{cc}A & 0 \\ 0 & B\end{array}\right)=p_{00}\left(\begin{array}{cc}A & 0 \\ 0 & B\end{array}\right)$. But since $\sigma\left(M_{C}\right)$ shrinks from $\sigma\left(\begin{array}{cc}A & 0 \\ 0 & B\end{array}\right)$, Lemma 2.2 gives

$$
\sigma\left(M_{C}\right) \backslash \omega\left(M_{C}\right) \subseteq p_{00}\left(\begin{array}{cc}
A & 0 \\
0 & B
\end{array}\right)
$$

Thus noting that iso $\sigma\left(\begin{array}{cc}A & 0 \\ 0 & B\end{array}\right) \subseteq$ iso $\sigma\left(M_{C}\right)$ passing to Lemma 2.1, it follows from $(0.3)$ that $\sigma\left(M_{C}\right) \backslash \omega\left(M_{C}\right) \subseteq p_{00}\left(M_{C}\right)$. The reverse is evident.

The condition "either $\mathcal{S P}(A)$ or $\mathcal{S P}(B)$ has no pseudoholes" is essential in Theorem 2.3. For example if $A$ and $B$ are operators on $\ell_{2} \otimes \ell_{2}$ defined by

$$
A=U \otimes 1 \quad \text { and } \quad B=U^{*} \otimes 1,
$$

where $U$ is the unilateral shift on $\ell_{2}$, then $\sigma\left(\begin{array}{cc}A & 0 \\ 0 & B\end{array}\right)=\omega\left(\begin{array}{cc}A & 0 \\ 0 & B\end{array}\right)$ and $p_{00}\left(\begin{array}{cc}A & 0 \\ 0 & B\end{array}\right)=\emptyset$, so that Browder's theorem holds for $\left(\begin{array}{cc}A & 0 \\ 0 & B\end{array}\right)$. But if the operator $C$ on $\ell_{2} \otimes \ell_{2}$ is defined by

$$
C=\left(\begin{array}{llll}
0 & & & \\
& 1-U U^{*} & & \\
& & 1-U U^{*} & \\
& & & \ddots
\end{array}\right),
$$


then a straightforward calculation shows

$$
\sigma\left(\begin{array}{cc}
A & C \\
0 & B
\end{array}\right)=\mathbb{D}, \quad \omega\left(\begin{array}{cc}
A & C \\
0 & B
\end{array}\right)=\mathbb{T} \quad \text { and } \quad p_{00}\left(\begin{array}{cc}
A & C \\
0 & B
\end{array}\right)=\emptyset
$$

which implies that Browder's theorem does not hold for $\left(\begin{array}{cc}A & C \\ 0 & B\end{array}\right)$.

On the other hand, Theorem 2.3 may fail for "Weyl's theorem" even with the additional assumption that Weyl's theorem holds for $A$ and $B$. To see this let the operators $A, B$ and $C$ on $\ell_{2}$ be defined by

$$
\begin{aligned}
& A\left(x_{1}, x_{2}, \cdots\right)=\left(0, x_{1}, 0, \frac{1}{2} x_{2}, 0, \frac{1}{3} x_{3}, 0, \frac{1}{4} x_{4}, \cdots\right) \\
& B\left(x_{1}, x_{2}, \cdots\right)=\left(0, x_{2}, 0, x_{4}, 0, x_{6}, 0, x_{8}, \cdots\right) \\
& C\left(x_{1}, x_{2}, \cdots\right)=\left(0,0, x_{2}, 0, x_{3}, 0, x_{4}, 0, \cdots\right):
\end{aligned}
$$

then

$$
\sigma(A)=\omega(A)=\{0\}, \quad \sigma(B)=\omega(B)=\{0,1\}, \quad \text { and } \quad \pi_{00}(A)=\pi_{00}(B)=\emptyset,
$$

which says that Weyl's theorem holds for $A$ and $B$. Also a straightforward calculation shows that

$$
\begin{aligned}
\sigma\left(\begin{array}{ll}
A & 0 \\
0 & B
\end{array}\right) & =\sigma\left(\begin{array}{ll}
A & C \\
0 & B
\end{array}\right)=\{0,1\}, \\
\omega\left(\begin{array}{ll}
A & 0 \\
0 & B
\end{array}\right) & =\omega\left(\begin{array}{ll}
A & C \\
0 & B
\end{array}\right)=\{0,1\}, \\
\pi_{00}\left(\begin{array}{ll}
A & 0 \\
0 & B
\end{array}\right) & =p_{00}\left(\begin{array}{ll}
A & 0 \\
0 & B
\end{array}\right)=\emptyset,
\end{aligned}
$$

while

$$
\pi_{00}\left(\begin{array}{cc}
A & C \\
0 & B
\end{array}\right)=\{0\} \neq p_{00}\left(\begin{array}{cc}
A & C \\
0 & B
\end{array}\right)=\emptyset
$$

which implies that Weyl's theorem holds for $\left(\begin{array}{cc}A & 0 \\ 0 & B\end{array}\right)$, but fails for $\left(\begin{array}{cc}A & C \\ 0 & B\end{array}\right)$. Note that Browder's theorem holds for $\left(\begin{array}{cc}A & C \\ 0 & B\end{array}\right)$.

We now have:

Theorem 2.4. If either $\mathcal{S P}(A)$ or $\mathcal{S P}(B)$ has no pseudoholes and if $A$ is an isoloid operator for which Weyl's theorem holds then for every $C \in \mathcal{L}(\mathcal{K}, \mathcal{H})$,

$$
\text { Weyl's theorem holds for }\left(\begin{array}{cc}
A & 0 \\
0 & B
\end{array}\right) \Longrightarrow \text { Weyl's theorem holds for }\left(\begin{array}{cc}
A & C \\
0 & B
\end{array}\right) \text {. }
$$

Proof. The same argument as the proof of Theorem 2.3 gives

$$
\sigma\left(M_{C}\right) \backslash \omega\left(M_{C}\right) \subseteq \pi_{00}\left(M_{C}\right)
$$


For the reverse inclusion of (2.4.1) suppose that $\lambda \in \pi_{00}\left(M_{C}\right)$. But if $\lambda \in \operatorname{iso} \sigma\left(M_{C}\right) \backslash$ iso $\sigma\left(\begin{array}{cc}A & 0 \\ 0 & B\end{array}\right)$ then, in view of Lemma 2.2, $\lambda$ should lie in $(\sigma(A) \cap \sigma(B)) \backslash \omega\left(\begin{array}{cc}A & 0 \\ 0 & B\end{array}\right)$, and hence $\lambda \notin \omega\left(M_{C}\right)$. Therefore it suffices to show that, for each $\lambda \in \pi_{00}\left(M_{C}\right) \cap$ iso $\sigma\left(\begin{array}{cc}A & 0 \\ 0 & B\end{array}\right)$,

$$
0<\operatorname{dim}\left(M_{C}-\lambda I\right)^{-1}(0)<\infty \Longrightarrow 0<\operatorname{dim}\left((A-\lambda I)^{-1}(0) \oplus(B-\lambda I)^{-1}(0)\right)<\infty \text { : }
$$

because (2.4.2) implies that if $\lambda$ is in $\pi_{00}\left(M_{C}\right) \cap$ iso $\sigma\left(\begin{array}{cc}A & 0 \\ 0 & B\end{array}\right)$ then $\lambda$ is in $\pi_{00}\left(\begin{array}{cc}A & 0 \\ 0 & B\end{array}\right)$, so that $\lambda \notin \omega\left(\begin{array}{ll}A & 0 \\ 0 & B\end{array}\right)=\omega\left(M_{C}\right)$ since Weyl's theorem holds for $\left(\begin{array}{cc}A & 0 \\ 0 & B\end{array}\right)$. For (2.4.2) suppose that $0<\operatorname{dim}\left(M_{C}-\lambda I\right)^{-1}(0)<\infty$. First of all observe that there is inclusion, in general,

$$
\left(M_{C}-\lambda I\right)^{-1}(0) \subseteq(A-\lambda I)^{-1}\left(C\left((B-\lambda I)^{-1}(0)\right)\right) \oplus(B-\lambda I)^{-1}(0),
$$

which forces that $(A-\lambda I)^{-1}(0) \oplus(B-\lambda I)^{-1}(0)$ is non-trivial because if it were not so then $\left(M_{C}-\lambda I\right)^{-1}(0)$ would be trivial, a contradiction. Now we must show that $(A-\lambda I)^{-1}(0) \oplus$ $(B-\lambda I)^{-1}(0)$ is finite dimensional. To the contrary we assume that $(A-\lambda I)^{-1}(0) \oplus(B-$ $\lambda I)^{-1}(0)$ is infinite dimensional. But since

$$
(A-\lambda I)^{-1}(0) \oplus\{0\} \subseteq\left(M_{C}-\lambda I\right)^{-1}(0),
$$

it follows that $\operatorname{dim}(A-\lambda I)^{-1}(0)<\infty$, so that $(B-\lambda I)^{-1}(0)$ must be infinite dimensional. Now there are two cases to consider.

Case 1. Suppose that $C\left((B-\lambda I)^{-1}(0)\right)$ is finite dimensional. Then $C^{-1}(0)$ must contain an orthonormal sequence $\left\{z_{j}\right\}$ in $(B-\lambda I)^{-1}(0)$. But then

$$
\left(\begin{array}{cc}
A-\lambda I & C \\
0 & B-\lambda I
\end{array}\right)\left(\begin{array}{c}
0 \\
z_{j}
\end{array}\right)=\left(\begin{array}{l}
0 \\
0
\end{array}\right) \quad \text { for each } j=1,2, \cdots,
$$

which implies that $\left(M_{C}-\lambda I\right)^{-1}(0)$ is infinite dimensional, a contradiction.

Case 2. Suppose that $C\left((B-\lambda I)^{-1}(0)\right)$ is infinite dimensional. Since

(i) $\lambda \in$ iso $\sigma\left(\begin{array}{cc}A & 0 \\ 0 & B\end{array}\right)$ and hence $\lambda \in \rho(A) \cup$ iso $\sigma(A)$;

(ii) $\operatorname{dim}(A-\lambda I)^{-1}(0)<\infty$;

(iii) $A$ is isoloid,

it follows that $\lambda \in \rho(A) \cup \pi_{00}(A)$. But since Weyl's theorem holds for $A$, we have that $A-\lambda I$ is Weyl, and hence $(A-\lambda I)(\mathcal{H})^{\perp}$ is finite dimensional. Therefore $C\left((B-\lambda I)^{-1}(0)\right) \cap$ $(A-\lambda I)(\mathcal{H})$ is infinite dimensional. Thus we can find an orthonormal sequence $\left\{y_{j}\right\}$ in $(B-\lambda I)^{-1}(0)$ for which there exists a sequence $\left\{x_{j}\right\}$ in $\mathcal{H}$ such that

$$
(A-\lambda I) x_{j}=C y_{j} \quad \text { for each } j=1,2, \cdots .
$$

But then

$$
\left(\begin{array}{cc}
A-\lambda I & C \\
0 & B-\lambda I
\end{array}\right)\left(\begin{array}{c}
x_{j} \\
-y_{j}
\end{array}\right)=\left(\begin{array}{l}
0 \\
0
\end{array}\right) \quad \text { for each } j=1,2, \cdots,
$$

which implies that $\left(M_{C}-\lambda I\right)^{-1}(0)$ is infinite dimensional, a contradiction. This completes the proof. 
The "isoloid" condition is essential in Theorem 2.4. For an example, consider the matrix $\left(\begin{array}{ll}A & C \\ 0 & B\end{array}\right)$, where $A, B$ and $C$ are given by $(2.3 .2),(2.3 .3)$ and (2.3.4), respectively: in fact, the operator $A$ in (3.2.2) is not isoloid. Also the condition "Weyl's theorem holds for $A$ " cannot be dropped in Theorem 2.4. For example if on $\ell_{2}$

$$
\begin{aligned}
& A\left(x_{1}, x_{2}, \cdots\right)=\left(0,0,0, \frac{1}{2} x_{2}, 0, \frac{1}{3} x_{3}, 0, \frac{1}{4} x_{4}, \cdots\right) ; \\
& B\left(x_{1}, x_{2}, \cdots\right)=\left(0, x_{2}, 0, x_{4}, 0, x_{6}, 0, x_{8}, \cdots\right) ; \\
& C\left(x_{1}, x_{2}, \cdots\right)=\left(x_{1}, 0, x_{2}, 0, x_{3}, 0, x_{4}, 0, \cdots\right):
\end{aligned}
$$

then the all the spectra are the same as (2.3.5) except

$$
\pi_{00}(A)=\{0\} .
$$

Therefore Weyl's theorem holds for $\left(\begin{array}{ll}A & 0 \\ 0 & B\end{array}\right)$, but fails for $\left(\begin{array}{ll}A & C \\ 0 & B\end{array}\right)$. Here note that Weyl's theorem does not hold for $A$, while $A$ is isoloid.

Corollary 2.5. If $A \in \mathcal{L}(\mathcal{H})$ is essentially normal isoloid operator for which Weyl's theorem holds then for every $C \in \mathcal{L}(\mathcal{K}, \mathcal{H})$,

$$
\text { Weyl's theorem holds for }\left(\begin{array}{cc}
A & 0 \\
0 & B
\end{array}\right) \Longrightarrow \text { Weyl's theorem holds for }\left(\begin{array}{cc}
A & C \\
0 & B
\end{array}\right) \text {. }
$$

Hence, in particular, if $A \in \mathcal{L}(\mathcal{H})$ is normal then Weyl's theorem is transmitted from $\left(\begin{array}{ll}A & 0 \\ 0 & B\end{array}\right)$ to $\left(\begin{array}{ll}A & C \\ 0 & B\end{array}\right)$ for every $C \in \mathcal{L}(\mathcal{K}, \mathcal{H})$.

Proof. The first assertion follows from Theorem 2.4 together with the fact ([20, Proposition 2.16]) that the spectral picture of every essentially normal operator has no pseudoholes. The second assertion follows at once from the first.

For an application of Corollary 2.5 we review a few facts concerning Toeplitz operators with continuous symbols (cf. [6]): if $\varphi \in C(\mathbb{T})$ then

$$
\begin{aligned}
& T_{\varphi} \text { is Fredholm if and only if } \varphi \text { is invertible; } \\
& \text { ind }\left(T_{\varphi}\right)=-w n(\varphi) \text { and } \sigma_{e}\left(T_{\varphi}\right)=\varphi(\mathbb{T}),
\end{aligned}
$$

where $w n(\varphi)$ denotes the winding number of $\varphi$ with respect to the origin.

Example 2.6. Suppose that $T_{\varphi}$ and $T_{\psi}$ are Toeplitz operators with continuous symbols $\varphi, \psi \in C(\mathbb{T})$ satisfying

$$
w n(\varphi-\lambda) w n(\psi-\lambda) \geq 0 \quad \text { for each } \lambda \in \mathbb{C} \backslash(\varphi(\mathbb{T}) \cup \psi(\mathbb{T})) .
$$

Then Weyl's theorem holds for $\left(\begin{array}{cc}T_{\varphi} & S \\ 0 & T_{\psi}\end{array}\right)$ with every $S \in \mathcal{L}\left(H^{2}\right)$. Hence, in particular, if $\varphi$ and $\psi$ are both analytic (or coanalytic) then Weyl's theorem holds for $\left(\begin{array}{cc}T_{\varphi} & T_{\varsigma} \\ 0 & T_{\psi}\end{array}\right)$ with every $\varsigma \in L^{\infty}(\mathbb{T})$.

Proof. Observe, by (2.5.1), (2.5.2) and (2.6.1), that $\omega\left(\begin{array}{cc}T_{\varphi} & 0 \\ 0 & T_{\psi}\end{array}\right)=\omega\left(T_{\varphi}\right) \cup \omega\left(T_{\psi}\right)$. Therefore by (1.0.1), Weyl's theorem holds for $\left(\begin{array}{cc}T_{\varphi} & 0 \\ 0 & T_{\psi}\end{array}\right)$. Further noting that, by (1.6.1), $T_{\varphi}$ and $T_{\psi}$ 
are essentially normal and that every Toeplitz operator is isoloid, it follows from Corollary 2.5 that Weyl's theorem holds for $\left(\begin{array}{cc}T_{\varphi} & S \\ 0 & T_{\psi}\end{array}\right)$ with every $S \in \mathcal{L}\left(H^{2}\right)$. For the second assertion note that if $\varphi$ and $\psi$ are both analytic (or coanalytic) then $w n(\varphi-\lambda) w n(\psi-\lambda) \geq 0$ for every $\lambda$ in each hole.

\section{Weyl's theorem for $2 \times 2$ commutative operator matrices}

When the entries in an operator matrix commute then the most of the familiar kinds of spectrum $\varpi$ can be calculated by determinants (cf. [9, Theorem XI.7.2],[13, Theorem 2.3]): if $\left(\begin{array}{ll}A & C \\ D & B\end{array}\right)$ is a commutative operator matrix on $\mathcal{H} \oplus \mathcal{H}$ then

$$
\varpi\left(\begin{array}{ll}
A & C \\
D & B
\end{array}\right)=\{\lambda \in \mathbb{C}: 0 \in \varpi((A-\lambda I)(B-\lambda I)-C D)\}
$$

Indeed (3.0.1) holds for the ordinary spectrum, the essential spectrum and the eigenvalues. We now have:

Theorem 3.1. Let $\left(\begin{array}{cc}A & C \\ 0 & B\end{array}\right)$ be a commutative operator matrix acting on $\mathcal{H} \oplus \mathcal{H}$. Then we have:

(a) Browder's theorem holds for $\left(\begin{array}{cc}A & 0 \\ 0 & B\end{array}\right)$ if and only if it holds for $\left(\begin{array}{cc}A & C \\ 0 & B\end{array}\right)$.

(b) Weyl's theorem holds for $\left(\begin{array}{cc}A & 0 \\ 0 & B\end{array}\right)$ if and only if it holds for $\left(\begin{array}{cc}A & C \\ 0 & B\end{array}\right)$.

Proof. Observe, by (3.0.1), that there is equality

$$
\varpi\left(\begin{array}{cc}
A & 0 \\
0 & B
\end{array}\right)=\varpi\left(\begin{array}{cc}
A & C \\
0 & B
\end{array}\right) \quad \text { for each } \varpi \in\left\{\sigma, \pi_{0}, \sigma_{e}, \omega\right\},
$$

where $\pi_{0}(\cdot)$ denotes the set of eigenvalues: the equality for $\omega$ follows from the fact that ind $\left(\begin{array}{cc}A-\lambda I & 0 \\ 0 & B-\lambda\end{array}\right)=$ ind $\left(\begin{array}{cc}A-\lambda I & C \\ 0 & B-\lambda I\end{array}\right)$ for every $\lambda \in \mathbb{C} \backslash \sigma_{e}\left(\begin{array}{cc}A & 0 \\ 0 & B\end{array}\right)$. We now claim that (3.1.1) also holds with $\varpi=p_{00}$ and with $\varpi=\pi_{00}$. In view of (3.1.1) it suffices to show

$$
\operatorname{dim}\left((A-\lambda I)^{-1}(0) \oplus(B-\lambda I)^{-1}(0)\right)<\infty \Longleftrightarrow \operatorname{dim}\left(M_{C}-\lambda I\right)^{-1}(0)<\infty .
$$

The forward implication follows from (2.4.3). For the backward implication suppose that $\operatorname{dim}\left(M_{C}-\lambda I\right)^{-1}(0)<\infty$. Then in view of $(2.4 .4)$, it suffices to show that $\operatorname{dim}(B-$ $\lambda I)^{-1}(0)<\infty$. To the contrary we assume that $(B-\lambda I)^{-1}(0)$ contains an orthonormal sequence $\left\{y_{j}\right\}$. Then since $C A=A C$, we have

$$
\left(\begin{array}{cc}
A-\lambda I & C \\
0 & B-\lambda I
\end{array}\right)\left(\begin{array}{c}
C y_{j} \\
(\lambda I-A) y_{j}
\end{array}\right)=\left(\begin{array}{l}
0 \\
0
\end{array}\right) \quad \text { for every } j=1,2, \cdots .
$$

Thus we must have that $\operatorname{dim}\left\{C y_{j}: j=1,2, \cdots\right\}<\infty$, and hence we can find an orthonormal sequence $\left\{z_{j}\right\}$ in $C^{-1}(0) \cap(B-\lambda I)^{-1}(0)$. But then

$$
\left(\begin{array}{cc}
A-\lambda I & C \\
0 & B-\lambda I
\end{array}\right)\left(\begin{array}{c}
0 \\
z_{j}
\end{array}\right)=\left(\begin{array}{l}
0 \\
0
\end{array}\right) \quad \text { for every } j=1,2, \cdots
$$


which implies that $\left(M_{C}-\lambda I\right)^{-1}(0)$ is infinite dimensional, a contradiction. This proves (3.1.2) and completes the proof.

When $\left(\begin{array}{ll}A & C \\ D & B\end{array}\right)$ is a commutative operator matrix we need not expect that Browder's theorem is transmitted from $\left(\begin{array}{cc}A & C \\ 0 & B\end{array}\right)$ to $\left(\begin{array}{ll}A & C \\ D & B\end{array}\right)$. For example Browder's theorem may fail for $\left(\begin{array}{cc}0 & C \\ C & 0\end{array}\right)$ (note that Weyl's theorem always holds for the nilpotent matrix $\left(\begin{array}{ll}0 & C \\ 0 & 0\end{array}\right)$ ): for a concrete example, take $C=T_{\varphi}$ as in Example 1.8.

\section{REFERENCES}

1. S.K. Berberian, An extension of Weyl's theorem to a class of not necessarily normal operators, Michigan Math. J. 16 (1969), 273-279.

2. S.K. Berberian, The Weyl spectrum of an operator, Indiana Univ. Math. J. 20 (1970), 529-544.

3. B. Chevreau, On the specral picture of an operator, J. Operator Theory 4 (1980), 119-132.

4. N.N. Chourasia, On Weyl's theorem for spectral operators and essential spectra of direct sum, Pure Appl. Math. Sci. 15 (1982), 39-45.

5. L.A. Coburn, Weyl's theorem for nonnormal operators, Michigan Math. J. 13 (1966), 285-288.

6. R.G. Douglas, Banach Algebra Techniques in the Operator Theory, Academic press, New York, 1972.

7. Hong-Ke Du and Jin Pan, Perturbation of spectra of $2 \times 2$ operator matrices, Proc. Amer. Math. Soc. 121 (1994), 761-766.

8. D.R. Farenick and W.Y. Lee, Hyponormality and spectra of Toeplitz operators, Trans. Amer. Math. Soc. 348 (1996), 4153-4174.

9. I. Gohberg, S. Goldberg and M.A. Kaashoek, Classes of Linear Operators (vol I), Birkhäuser, Basel, 1990.

10. P.R. Halmos, Hilbert Space Problem Book, Springer, New York, 1984.

11. R.E. Harte, Fredholm, Weyl and Browder theory, Proc. Royal Irish Acad. 85A (2) (1985), 151-176.

12. R.E. Harte, Invertibility and Singularity for Bounded Linear Operators, Dekker, New York, 1988.

13. R.E. Harte, Invertibility and singularity of operator matrices, Proc. Royal Irish Acad. 88A (2) (1988), $103-118$.

14. R.E. Harte and W.Y. Lee, Another note on Weyl's theorem, Trans. Amer. Math. Soc. 349 (1997), $2115-2124$.

15. R.E. Harte, W.Y. Lee and L.L. Littlejohn, On generalized Riesz points (to appear).

16. W.Y. Lee, Weyl spectra of operator matrices (to appear).

17. W.Y. Lee and S.H. Lee, A spectral mapping theorem for the Weyl spectrum, Glasgow Math. J. 38(1) (1996), 61-64.

18. K.K. Oberai, On the Weyl spectrum, Illinois J. Math. 18 (1974), 208-212.

19. K.K. Oberai, On the Weyl spectrum (II), Illinois J. Math. 21 (1977), 84-90.

20. C.M. Pearcy, Some Recent Developements in Operator Theory, CBMS 36, Providence:AMS, 1978.

21. C. Schmoeger, Ascent, descent and the Atkinson region in Banach algebras II, Ricerche di Matematica vol. XLII, fasc. $2^{\circ}$ (1993), 249-264.

22. H. Weyl, Über beschränkte quadratische Formen, deren Differenz vollsteig ist, Rend. Circ. Mat. Palermo 27 (1909), 373-392.

23. H. Widom, On the spectrum of a Toeplitz operator, Pacific J. Math. 14 (1964), 365-375.

Department of Mathematics

Sungkyunkwan University

Suwon 440-746, Korea

E-mail: wylee@yurim.skku.ac.kr

1991 Mathematics Subject Classification. Primary 47A53, 47A55 